\title{
PAPEL DEL LOGOPEDA EN UN CENTRO GERONTOLÓGICO DE ESTANCIAS DIURNAS
}

\author{
L. Lodeiro Fernández a , N. Varela González ${ }^{\text {, A. A. López Sande }}$, M. Gandoy Crego ${ }^{\mathrm{d}}$ y J. \\ Millán Calenti ${ }^{\mathrm{e}}$ \\ ${ }^{a}$ Logopeda experta en Gerontología Clínica. ${ }^{b}$ Enfermera experta en Gerontología Clínica. ${ }^{c}$ Psicóloga Clínica. \\ Master en Gerontología Clínica. ${ }^{d}$ Doctor en Psicología. Master en Gerontología Clínica y Social. ${ }^{e}$ Catedrático (EU) \\ Gerontología Clínica y Social. Director grupo de Investigación en Gerontología. Departamento de Medicina. \\ Universidad de A Coruña. Grupo Investigación Gerontología. Departamento de Medicina. Universidad de La \\ Coruña.
}

\section{RESUMEN}

El aumento de población anciana en nuestra sociedad es un hecho que ha provocado la aparición de nuevos recursos socio-sanitarios que cubran sus necesidades. El Centro Gerontológico de Estancias Diurnas es un servicio multidisciplinar diurno que se ocupa de la atención social y sanitaria, con actuación terapéutica y preventiva, de los mayores facilitando la vida laboral de las personas que se ocupan ordinariamente de su cuidado.

Los centros de día deben proporcionar una atención integral debiendo, por tanto, estar constituidos por un equipo profesional multidisciplinar en el que la figura del logopeda esté presente desarrollando una labor específica. Parece, por tanto, interesante delimitar las funciones básicas a desempeñar, los trastornos más comunes susceptibles de intervención, así como, algunas directrices a tener en cuenta.

Palabras clave: Logopedia, Centro Gerontológico de Estancias Diurnas, envejecimiento.

\section{SUMMARY}

The increase of the senior population in OUJ society is an event that has provoked the appeareance of a new sort of social and sanitary resource, a structure that has to be concerned with the needs of this population. The gerontologic day-center is in brief a multidisciplinar service, that works during day time, treating the social and sanitary occupation of our elder with both therapeutic and preventive acts. This to provide a normal working life for the people who are usually taking care of these people. Day-centers have to answer these needs with a complete service, and for that rea on they have to apile to a multidisciplinar staff in wich the speech-therapist develops a specific role It seems though interesting to define the basic functions that a professional speech-therapist has to perform, such as the most common disorders capable of treatment and general intervention, as well derectives to bear in mind.

Key words: Speech therapy, Gerontology Center for Daytime Stay, ageing. 


\section{INTRODUCCIÓN}

Definimos el Centro Gerontológico de Estancias Diurnas (CGED) como un recurso multiasistencial, de carácter rehabilitador, dirigido a personas mayores que presenten alguna problemática de tipo socioasistencial y configurado como una estructura de atención diurna que proporciona asistencia continuada durante tiempo indefinido, de manera que posibilita la vida socio-laboral del resto de miembros de la familia, al cuidado del paciente.

Dentro de esta definición, se excluyen los hogares y clubs de pensionistas y jubilados cuya característica principal es facilitar el ocio y fomentar actividades culturales entre sus socios y los centros de día con ubicación independiente o integrados dentro de una residencia, en donde sus fines no son siempre administrar cuidados o terapia rehabilitadora, por lo que en muchos casos han sido denominados vulgarmente como "geroguarderías", ya que en ellos se dejan los pacientes al cuidado de personal casi siempre auxiliar (Buide, 2000), quedando la denominación, por nosotros propuesta de Centros Gerontológicos de Estancias Diurnas para aquellos centros cuya actividad principal, por ser "gerontológicos" es la rehabilitadora sobre el usuario y de apoyo a las familias y que reúnan las siguientes características diferenciadoras:

- Disponer de Unidad de Transporte Adaptada.

- Disponer de sala de rehabilitación independiente de la sala polivalente.

- Tener a sus profesionales sanitarios integrados en equipos multidisciplinares.

- Disponer de personal específico de rehabilitación.

- Trabajar mediante protocolos de intervención.

Se configuran, por tanto, los CGED como una alternativa de atención socio-sanitaria dirigida a aquellas personas mayores que presentan algún tipo de dependencia, de carácter leve o moderado, bien física, bien psíquica, bien mixta; siendo, por tanto, muchos los cuidados a dispensar y los objetivos a alcanzar, entre los que estará evitar la institucionalización permanente de los pacientes, facilitando la permanencia en su entorno habitual con lo que mantendrán el contacto con su familia, a la vez que tiene la posibilidad de visitar al paciente.

Durante la permanencia del paciente en el CGED, tras proceder a la valoración general de su situación bio-psico-social y funcional, se pondrán en marcha todas las medidas de carácter preventivo y asistencial necesarias para lograr mantenerle en la mejor situación referida a su calidad de vida, todo sin olvidarnos de la familia, a la que se le ofrecerá formación e información acerca del proceso que aqueja al paciente y la disponibilidad de acciones de intervención de acuerdo a las características del centro, que en todo caso ha de contar con un personal básico, formado en el campo de la gerontología y geriatría y que incluiría al médico rehabilitador, al psicólogo clínico, al fisioterapeuta, al terapeuta ocupacional, al logopeda, a la enfermera y al trabajador social como personal más cualificado, a los que se unirían los gerocultores o auxiliares, que serían el personal encargado de dispensar los cuidados básicos en las actividades de la vida diaria; es decir, higiene, alimentación, continencia y movilización.

El equipo gerontológico, tras la valoración de cada uno de los usuarios, pondrá en marcha los protocolos de intervención específicos, que han de incluir las adecuadas medidas potenciadoras, mantenedoras o bien recuperadoras de los déficits potenciales, siempre actuando de manera personalizada (Arriola, 2000).

A fin de facilitar el desplazamiento de los pacientes, los CGED deberán de disponer de una Unidad de Transporte Adaptada, que será la encargada de recoger a los usuarios que no puedan desplazarse por sus propios medios al centro, para que una vez finalizada la estancia, reubicarlos en su domicilio; de esta manera se cumple un doble objetivo, por un lado, que personal especializado apoye a las familias en la movilización del usuario durante el transporte desde su domicilio, mientras que por otro, la recogida en el domicilio dará más flexibilidad a la familia o cuidadores de la red natural en cuanto a no estar pendientes de disponer de medios para un transporte que a veces es complicado, sobre todo si hablamos de pacientes que se desplazan en sillas de ruedas. 


\section{EL LOGOPEDA}

El logopeda como miembro del equipo gerontológico del centro, y aunque con la función principal de evaluar e intervenir sobre los trastornos de la comunicación: lenguaje, habla, voz y audición, deberá de participar en otras actividades propias de todo personal sanitario, como son la estimulación del lenguaje oral y escrito con otros usuarios del centro aún sin patología lingüística (Acuña, 1997), a fin de preservar sus capacidades, y caso de presentar un cuadro demencial en fases iniciales, retrasar lo más posible la pérdida de su capacidad comunicativa; participar en la educación sanitaria, informando a los familiares, cuidadores y profesionales de las características del trastorno detectado y de las medidas de mejora, facilitando, además, consejos sobre la comunicación de los que puedan beneficiarse las personas del entorno de los pacientes, especialmente cuando nos referimos a pacientes con demencia, con lo que se conseguirá mejorar la adaptación emocional y social de los implicados.

También el logopeda intervendrá en el fomento de relaciones sociales entre los usuarios del centro, a fin de incrementar los "momentos comunicativos", que a su vez contribuirán al mantenimiento de las capacidades comunicativas y actuará fomentando las habilidades sociales en el campo de la comunicación (Caballo, 1993), En pacientes donde la alteración que presenten afecte a la comunicación, aplicará sistemas alternativos a fin de facilitarla (Basil, 1998) y trabajará en el campo de la prevención enseñando técnicas que permitan el cuidado de la voz, tanto en lo que se refiere a los pacientes, como a las familias y al personal del centro.

La detección de trastornos comunicativos es otra de las funciones que el logopeda deberá de realizar, detectando el posible origen o enfermedad de base que lo provoca, así en las primeras fases de la enfermedad de Alzheimer, pue- de ser difícil distinguir los cambios que afectan a la comunicación de los generados por el propio proceso de envejecimiento, en donde los límites entre lo patológico y lo fisiológico no están muy definidos.

Dentro de la problemática de las personas que potencialmente serán los usuarios de un CGED, es fácil encontrar alteraciones de la deglución, en base a trastornos físicos o psíquicos, campo en el que el logopeda también deberá de intervenir.

Por otro lado sabemos que la realización eficaz de las tareas conducentes a mejorar la comunicación van a suponer una reducción de la carga del cuidador, por ser la comunicación una de las actividades básicas cuya pérdida más va a influir en la aparición de ansiedad por parte del cuidador como ya se ha puesto de manifiesto por diferentes autores (Ravins, 1982; Millán, 2000), que establecen un incremento del tiempo de dedicación al paciente en base al deterioro de la capacidad de lenguaje; así un paciente con trastornos de comprensión no va a entender frases largas ni complejas, con lo que la intervención sobre la capacidad comunicativa del paciente, a la vez que sobre la técnica de lenguaje del cuidador, permitirá una comunicación más fluida, a la vez que más relajada.

En resumen, la intervención del logopeda en un CGED se va a referir a personas con características diferenciadoras: sin deterioro, con alteraciones leves, moderadas o graves de la comunicación; y pautadas desde enfoques, también, muy diferentes tanto desde el punto de vista oral, como de los sistemas aumentativos.

Por último, y por su importancia, deberemos de referirnos a la investigación, como otra de las actividades que el logopeda deberá de incluir dentro de su devenir diario, ya que a través de ella, a la vez que podrá contestar a las preguntas metodológicas que puedan surgirle, podrá realizar un control y seguimiento de los pacientes y protocolos que está desarrollando y de esta manera conocer su grado de efectividad. En este sentido, aunque el logopeda realizará una serie de funciones específicas de él, como miembro del equipo multidisciplinar, deberá de participar en todas las líneas y proyectos de investigación que con el objetivo de mejorar la calidad asistencial se pongan en marcha en su centro, además de divulgarlas a través de los medios científicos para el conocimiento de la sociedad.

\section{Bases de actuación}

En cuanto a las bases de actuación del logopeda en un CGED, tras el ingreso del paciente y en base al informe médico, procederá a realizar la valoración específica pertinente, según el tipo de trastorno, para a continuación establecer un "plan de intervención específico", que integrado en el general, incluirá unos objetivos a conseguir. Dichos objetivos, han de estar adaptados a las características de las personas 
mayores, en donde en muchas ocasiones, más que la recuperación total de la función, no factible, se ha de pensar en cómo preservar las habilidades que se mantienen o cómo paliar los déficits.

En algunas situaciones, ante determinadas alteraciones, o en estadíos evolucionados de la enfermedad o por la presencia de trastornos invalidantes, puede no estar indicado iniciar la terapia, como es el caso de un enfermo de Alzheimer en fase terminal; o retrasarla, por presentar el paciente un trastorno depresivo que lo desmotive. En estos casos, las posibilidades de mejoría son nulas y los trastornos que se van a generar al paciente van a ser peores que los resultados a conseguir, estando indicado entonces intervenir sobre los cuidadores y demás personas del entorno para mejorar la comunicación, por ejemplo, no verbal.

Se establecen, entonces, en función de las expectativas de mejora, de acuerdo al pronóstico, diferentes posibilidades de actuación, así en algunos casos podremos conseguir la rehabilitación de la alteración, bien de manera total o parcial; en otros únicamente lograremos preservar las funciones durante un tiempo más o menos largo o tratar de paliar y no curar, buscando alternativas funcionales a los déficits generados mediante ayudas técnicas; y por último, en referencia a aquellos en que las posibilidades de actuación sobre el paciente son mínimas, entonces se actuará sobre el entorno facilitando la integración y el fomento de las relaciones sociales. Por supuesto, y dado que la rehabiltación es un concepto integral, en ningún caso las intervenciones han de ser excluyentes, sino más bien complementarias, así en un paciente que sabemos que podemos rehabilitar una función, deberemos de contemplar las otras pautas de intervención sobre otras alteraciones, en cuanto a preservar funciones, paliar déficits, usar ayudas técnicas o actuar sobre el entorno.

El modelo de intervención con las personas mayores, hace necesario tener en cuenta una serie de características referidas a este colectivo en cuanto a la preservación de su capacidad funcional, necesaria para el mantenimiento de su independencia y por tanto para lograr un nivel de satisfacción elevado; el evitar la fatiga, mediante el fraccionamiento de las sesiones de terapia, teniendo en cuenta que de acuerdo a la pluripatología que les caracteriza, pueden ser varios los tratamientos que reciban a un tiempo, que padezcan dolor o, simplemente, que les cueste un mayor esfuerzo en relación a su discapacidad, en relación a otras personas más jóvenes, en donde los resultados satisfactorios se pueden conseguir antes; el ser pacientes y amables teniendo en cuenta que con la edad la capacidad de respuesta esta enlentecida y que por tanto las sesiones serán más largas; el tratar de ser cariñosos y afectuosos con él, ya que en ocasiones va a ser mejor terapia que la propia intervención; y por último, tener en cuenta la aparición concomitante de otros trastornos de puedan dificultar la intervención como pueden ser los trastornos cognitivos, alteraciones sensoriales o déficits motores.

En un CGED, muchas de las actuaciones logopédicas se harán de forma grupal: en base al número de pacientes que hay que atender y que pueden superar nuestra capacidad de dedicación en tiempo; a que sean menos fatigantes para los pacientes a los que dedicamos menos atención de manera individualizada y de esta forma se sienten más relajados y también por el hecho de fomentar nuevas relaciones dentro de la dinámica del centro.

Por otro lado, los materiales que vamos a utilizar, tanto para la evaluación como para la intervención no deberán de ser infantiles, sino para adultos y caso de ser necesario adaptados: aumentando el tamaño, potenciando los colores y utilizando imágenes reales, no abstractas, que a la vez sean manipulables.

\section{INDIVIDUOS SUSCEPTIBLES DE INTERVENCIÓN LOGOPÉDICA}

A un CGED, van a acudir pacientes mayores dependientes físicos o psíquicos, con diferentes patología, algunas propias de la intervención por parte del logopeda como son: las afasias y las disartrias, aisladas o propias de los cuadros demenciales; las disglosias, debidas a alteraciones de la cavidad bucal, principalmente, glosectomías y pacientes edéntulos; las alteraciones de la voz causadas por laringuectomías, las disfonías y las parálisis laríngeas; los déficits auditivo s bien como hipoacusias, bien generados por el envejecimiento (presbiacusias); los trastornos de deglución y los pacientes mayores sin patología lingüística pero en donde podremos realizar estimulación del lenguaje.

En relación al riesgo de sufrir enfermedad vascular cerebral por las personas mayores, la afasia es una de las complicaciones más frecuentes que presentan estos enfermos, reflejando los estudios (Chipps, 1995) que hasta un $72 \%$ de los ACV se producen en mayores de 65 años y de ellos el $40 \%$ desarrollan una afasia (Pérez-Pamies, 1992). Aunque algunos estudios reflejan que la edad es un factor pronóstico negativo (Ducarne, 1989), siempre que el estado físico-psíquico-psicológico del paciente sea el adecuado debe iniciarse la rehabilitación. 
Es necesario conocer la progresión del deterioro lingüístico propio del envejecimiento, a fin de distinguirlo del patológico que aparece en el desarrollo de los cuadros demenciales (Brosa, 1999), además, dentro de cada tipo de demencia se observarán particularidades en cuanto al deterioro lingüístico (Helm-Estabrooks, 1994; Juncos, 1998). En general, las demencias que originan afasia son las de tipo cortical como la enfermedad de Alzheimer o la enfermedad de Pick (Alberca, 1999), siendo la enfermedad de Alzheimer la forma más frecuente de demencia (50-60 \% de los casos), seguida de las demencias vasculares (15-30 \%).

La evaluación del lenguaje se realizará conjuntamente con la valoración cognitiva realizada por el psicólogo del centro, para a continuación iniciar la intervención por grupos homogéneos, sin olvidarse del lenguaje escrito (lectura y escritura) de acuerdo a las características del mismo en estos niveles de deterioro (González, 1993). Los objetivos y las expectativas de intervención son diferentes a los casos de afasia sin demencia: no se trata de rehabilitar las funciones perdidas, sino de retardar el proceso de deterioro, manteniendo las habilidades presentes y compensando los déficits. Teniendo en cuenta estas consideraciones, la rehabilitación de la afasia en las personas mayores, de manera general, ha de seguir las normas y el método de la rehabilitación en los adultos, mediante la enseñanza programada basada en el condicionamiento operante postulado por Skinner, en donde se establece una jerarquización del material a aprender, descomponiéndolo en unidades, en las que unas son sustento de otras posteriores y en dónde el éxito de la respuesta será un refuerzo positivo, que supone avance, mientras que el fracaso, supondrá la necesidad de seguir afianzando el aprendizaje en ese estadío o, incluso, el retroceso a estadíos anteriores. Se utilizarán sistemas alternativos de comunicación: para casos severos en los que no sean viables las terapias verbales y actividades de estimulación cognitiva. El MIT (Melodic Intonation Therapy) desarrollado pro Albert, Sparks y Helm (1973), pretende hacer surgir la expresión oral a partir del apoyo en la melodía en pacientes donde fracasan los métodos tradicionales. Los medios audiovisuales y la rehabilitación en grupo, se utilizarán como complemento de la rehabilitación individual.

La intervención de la afasia, por tanto, tiene como base todos estos principios, abarcando la reeducación en todos aquellos aspectos del lenguaje que se vean afectados y que podría resumirse en: Reeducación de los trastornos de la expresión verbal: reducción del débito y de la jerga logorreica, trastornos fonéticos y fonológicos, alteraciones lexicales y alteraciones sintácticas; Reeducación de los trastornos de la comprensión verbal: trastorno gnósico de la comprensión verbal, trastorno psicolingüístico de la comprensión oral, alteración en la retención de información auditiva o fallo acústico-amnésico y alteración por falta de atención. Reeducación de la alexia y agrafia. (Pérez-Pamies, 1994).

La importancia de las actividades reside en la ejercitación de los procesos lingüísticos más que en el número de aciertos del paciente. Se evitará la sobrecarga y frustración del paciente. La estimulación del lenguaje se complementará con la estimulación cognitiva general, siguiendo la técnica de la Terapia de Orientación a la Realidad (Salom, 2000). No hay que olvidar que esta afasia es una consecuencia de un deterioro cognitivo generalizado. Por eso, algunos autores diferencian el trastorno del lenguaje de una afasia aislada del de una afasia con demencia especificando que la afasia es una alteración que afecta específicamente al sistema lingüístico, debida a lesión cerebral focal, y que puede ir acompañada de otras alteraciones cognitivas. En la demencia las alteraciones del lenguaje, que pueden ser parecidas a las de la afasia, son la manifestación de alteraciones en otros sistemas cognitivos (Juncos, 1993), pudiendo considerarse el trastorno más comunicativo que lingüístico, por lo que la intervención deberá de hacer más énfasis en ese aspecto: una forma sería preocupándose de que el entorno propicie el mantenimiento de la comunicación el máximo tiempo posible (Pierre, 1994a).

Por otro lado, en cuanto a las disartrias, estas pueden ser: de tipo extrapiramidal como en la enfermedad de Parkinson o las cerebelosas; piramidal por afectación pseudobulbar como la de la desintegración lacunar o la de la esclerosis en placas; de la primera neurona como en la esclerosis lateral amiotrófica y la de la parálisis bulbar progresiva; y secundarias a encefalopatías seniles como las de las demencias subcorticales tipo demencia Parkinsoniana o Corea de Huntington.

La enfermedad de Parkinson, con una incidencia de unos 130 casos por 100.000 sujetos, aumenta su prevalencia con la edad considerándose, incluso, como el proceso neurológico más frecuente entre los mayores de 60 años (Chipps, 1995); la evolución degenerativa y progresiva de la enfermedad varía de un paciente a otro, aunque el 10-15\% de los afectados desarrollan un cuadro de demencia. Cuando el paciente presenta disartria, el tratamiento será el convencional, teniendo en cuenta las características de la persona mayor en cuanto a la forma de intervenir, como ya se ha reflejado anteriormente, fundamentándonos en la enseñanza de técnicas de respiración, fonación, resonancia, articulación y 
prosodia, que ayuden a potenciar las facultades del habla. Cuando el trastorno está en fases avanzadas, una opción son los sistemas alternativos de comunicación.

La disartria con demencia es irreversible, por lo que la intervención ha de ir encaminada al mantenimiento de la máxima funcionalidad y a la potenciación de los efectos preservados que permitan compensar los déficits.

Las disglosias más frecuentes en los paciente mayores son las debidas a glosectomías, a la falta de piezas dentarias, principalmente de incisivos y a la utilización de prótesis mal adaptadas, sobre todo teniendo en cuenta que más de un 80 \% de los ancianos precisan prótesis (Arlette, 1999).

El resultado de la falta de dentadura es un cambio de los puntos de articulación, con la aparición de hipotonía en los músculos de las mejillas, labios y lengua provocando una voz débil Y nasalizada. La solución será la adaptación de una prótesis o bien la reeducación del habla en caso de intolerancia a la prótesis (Pierre, 1994b).

La intervención en las disglosias en general, se fundamenta en los principios de la terapia miofuncional que se puede definir como la terapia muscular facial realizada para la mejora de las funciones orofaciales. En cuanto a los trastornos específicos del habla de estos casos, la rehabilitación implica la enseñanza de los nuevos puntos y modos articulatorios de los fonemas que debido a las modificaciones en la cavidad bucal se hayan visto afectados, así como la enseñanza de técnica vocal que incida en la resonancia y por tanto mejore la alteración de voz que puede ocasionarse.

En pacientes laringuectomizados, se puede comenzar el aprendizaje de la voz erigmofónica (Perelló, 1973; Le Huche, 1982), además de establecer grupos de apoyo con otros pacientes en las mismas condiciones. En caso de negación se puede optar por el empleo de un sistema alternativo de comunicación, además de que la fisioterapia respiratoria ha de ser una técnica complementaria, junto con el empleo de prótesis laríngeas, caso de ser necesarias (De la Fuente, 1994).

Las alteraciones de la voz más comunes en los pacientes ancianos que puedan beneficiarse de la intervención en un CGED incluyen las disfonías, variables en su tipología al igual que en los adultos; las parálisis laríngeas y las afecciones propias de la edad (Juncos, 1998). El tratamiento será común y consiste en la enseñanza de la técnica vocal que implica el aprendizaje de los métodos adecuados de postura corporal, relajación en casos de tensión muscular, respiración, resonancia e impostación, principalmente. El tratamiento de las disfonías por parálisis laríngeas también se fundamentan en estas técnicas pero han de tener en cuenta métodos de potenciación de la musculatura de la hemilaringe parética, algunos autores como Arias (1994) indican de modo más específico el tipo de ejercicios a realizar. En el caso de las alteraciones fisiológicas debidas al envejecimiento natural del sistema fonatorio, pueden compaginarse con actividades de canto, individual o en coros, que a la vez facilitarán la socialización entre los usuarios.

Las dificultades auditivas en la vejez pueden ser debidas a dos factores: la disminución del campo auditivo y el enlentecimiento en el procesamiento de la información auditiva. En el primero de los casos, el logopeda recomendará el empleo de prótesis auditiva (audífono) y realizará la adaptación a la misma (Martínez, 1994). Consiste en asegurarse de que la prótesis auditiva cubre los déficits auditivos del paciente, realizando un seguimiento y control de eficacia. En caso necesario, iniciar un entrenamiento auditivo. Si no fuera posible la prótesis, se usarán métodos alternativos de comunicación, o completar la información con comunicación no verbal. En cuanto al enlentecimiento en el procesamiento de la información auditiva, propio de la edad, puede solucionarse si al dirigirnos al anciano se le habla despacio, de tal modo que procese la información al mismo tiempo que se le transmite. El logopeda debe informar a las personas de su entorno de esta medida.

Las disfagias pueden clasificarse según su naturaleza sea orofaríngea o esofágica, siendo la etiología diferente, así para las orofaríngeas, son causas los ACV, los síndromes bulbares y pseudobulbares, los traumatismos craneales, la enfermedad de Parkinson, la esclerosis en placas o lateral amiotrófica, las lesiones periféricas, las enfermedades musculares y los factores lesionales locales (infección, úlcera, tumor), mientras que para las esofágicas lo son los tumores, las inflamaciones o las comprensiones extrínsecas.

Las disfagias esofágicas no requieren tratamiento rehabilitador. La reeducación en los casos de disfagia orofaringea podrá retrasar la colocación de una sonda nasogástrica (Roser, 2000) y consiste en la enseñanza del control motor de la cabeza, mandíbula y lengua; corrección postural; elección de los 
alimentos en función de la evolución de las alteraciones; maniobras facilitadoras; estimulación de la sensibilidad oral; inhibición de reflejos arcaicos y ejercicios de vocalización para mejora del cierre glótico (Canu y Pierre, 1994).

Como hemos señalado a lo largo del artículo, incluso los ancianos sin patología comunicativa concreta pueden padecer algún tipo de alteración lingüística como las anomias, dificultades comprensivas de términos abstractos o de complejidad sintáctica, dificultades en narraciones y descripciones, "perder el hilo de la conversación", etc., por ello, mediante la estimulación que incida en esos aspectos puede retrasarse su deterioro e incluso retardar la aparición de la sintomatología lingüística, caso de que el paciente esté en la fase de inicio de la enfermedad de Alzheimer, similar, en todo caso, al del envejecimiento normal, pero con una base orgánica seguramente diferente (Jódar, 1992). La estimulación del lenguaje debería de contemplarse como una parte de la estimulación cognitiva, algunos autores como Martínez (2000) y como nosotros, la hemos desarrollado en nuestros programas de estimulación de la memoria con mayores. La experiencia adquirida tras la aplicación de un programa de estimulación de la memoria y del lenguaje en pacientes sin deterioro cognoscitivo, nos ha confirmado que su estimulación influye positivamente en la conservación de estas facultades.

\section{CONCLUSIONES}

El progresivo envejecimiento de la población, así como el incremento de las patologías edad dependientes, entre las que incluimos las demencias, los accidentes cerebro-vasculares, el parkinson, los tumores, las alteraciones sensoriales y otras muchas, con base principalmente neurológica o de otro tipo, hace que de un tiempo a esta parte se estén incrementando los recursos asistenciales dirigidos a los mayores, a la vez que los profesionales que se incorporan a ellos, sobre todo, cuando su carácter es eminentemente rehabilitador, pertenezcan a diferentes campos de las Ciencias de la Salud y/o Sociales, faltando en muchos casos el profesional de la logopedia como miembro de estos equipos multidisciplinares, a pesar de la gran cantidad de alteraciones de la vejez que cursan con trastornos del lenguaje. La actuación logopédica en el campo de la gerontología está debidamente fundamentada y aunque pueda parecer innovadora, lejos de ello, debiera de estar justificada desde el momento que hay que intervenir sobre la comunicación, tanto cuando esta se refiere al paciente, como cuando lo hace a la familia o los allegados, sin olvidar el papel de terapeuta que el logopeda debe de tener en la corrección de las anomalías detectadas en el resto de los profesionales que trabajan junto a él. Además, como en el resto de las Ciencias de la Salud aplicadas, la prevención es la principal arma con que contamos a fin de evitar el trastorno o las secuelas, de ahí que en este campo el logopeda también tiene mucho que aportar. Pero todo ello ha de basarse en un buen conocimiento de su rol, junto con la especialización en las técnicas de aplicación a los mayores, de manera complementaria al conocimiento de las características psico-sociales de este colectivo.

Por último, concluir que cualquier actividad ha de basarse en la formación, en la experiencia y por supuesto en la investigación a fin de incorporar los adelantos al momento de la intervención y que ésta sea de la mayor calidad.

\section{BIBLIOGRAFÍA}

Acuña M, Risiga M. Talleres de activación cerebral y entrenamiento de la memoria. Barcelona: Paidós, 1997.

Alberca R, López-Pousa S. Enfermedad de Alzheimer y otras demencias. Editorial Médica Panamericana, 1999;75-9.

Arias Marsal C. Parálisis laríngeas: diagnóstico y tratamiento foniátrico de las parálisis cordales unilaterales en abducción. Barcelona: Masson, 1994.

Arlette Pinzón S, Zunzunegui V. Detección de necesidades de atención bucodental en ancianos mediante la autopercepción de la salud oral. Rev Mult Gerontol 1999;9:216-24.

Arriola Manchola E. Recursos sociosanitarios-EI centro de día (CD) psicogeriátrico. Rev Mult Gerontol 2000;10(2):105-25.

Basil Almirall C, Soro-Camats E, Roscell Bultó C. Sistemas de signos y ayudas técnicas para la comunicación aumentativa y la escritura. Barcelona: Masson, 1998.

Brossa Olacinegui J, Martínez Herrador JL. Trastornos en la denominación en los pacientes con la enfermedad de Alzheimer: estudio comparativo. Geriátrica 1999;15(6):284-9.

Buide AM, Fernández E, Yarela N, Millán JC, Gandoy M, Mayán JM. Papel del profesional de enfermería en un centro de día Gerontológico. Gerokomos 2000; 11: 133-7.

Caballo V. Manual de Evaluación y Entrenamiento de las Habilidades Sociales. Madrid: Siglo XXI, 1993.

Canu S, Pierre J. Tratamiento de los trastornos de la deglución. En: Thévenon A, Pollez B, eds. Rehabilitación en geriatría. Barcelona: Masson, 1994;92-3. 
Chipps E, Clanin N, Campbell V. Trastornos neurológicos, Madrid: Mosby/Doyma Libros, 1995;68-147,

De la Fuente Gonzá1ez M, En: Parreño Rodríguez JR, ed. Rehabilitación en geriatría, 2ª ed. Madrid: Editores Médicos, 1994;349.

Ducarne de Ribacourt B. Reeducación semiológica de la afasia. Barcelona: Masson, 1989;188-93.

Gonzá1ez Montalvo JI, Del Ser T, Bermejo F. Creación y validación de un test de lectura para el diagnóstico del deterioro mental en el anciano. En: Bermejo F, ed. Nivel de salud y deterioro cognitivo en los ancianos. Barcelona, 1993;143-5.

Helm-Estabrooks N, Martín LA. Manual de terapia de la afasia. Madrid: Ed. Médica Panamericana, 1994;70-2.

Jódar Vicente M. Envejecimiento normal versus demencia de Alzheimer. Valor del1enguaje en el diagnóstico diferencial. Rev Logop Fon Audiol 1992;XII(3):171-9.

Juncos Rabadán O. Consideraciones sobre el1enguaje y la intervención logopédica en la tercera edad. Rev Logop Fon Audiol 1993;XIII(2):79-88.

Juncos Rabadán O. Lenguaje y envejecimiento: bases para la intervención. Barcelona: Masson, 1998;73-94.

Le Huche F. La voz sin laringe (manual de reeducación vocal). Barcelona: Médica y Técnica, 1982.

Martínez C. Haciendo memoria: estimulación de las funciones mentales en las personas mayores. Guipúzcoa: Oria, 2000.

Martínez J. Prótesis auditivas. En: Peña Casanova, ed. Manual de logopedia, 2ª ed. Barcelona: Masson, 1994;210-27.

Millán JC, Gandoy M, Antelo M, Mayán M. Supervisión nocturna, diurna y comunicación; tres nuevos ítems para valorar la carga asistencial del paciente con deterioro cognitivo. Rev Mult Gerontol 2000;10(1):20-5.

Pere1ló J. Alteraciones de la voz. Barcelona: Científica y Médica, 1973.

Perez-Pamies M. Afasias del adulto. En: Peña-Casanova J, ed. Manual de logopedia, $2^{\text {a }}$ ed. Barcelona: Masson, 1994;305.

Pierre J. Logopedia. En: Thévenon A, Pollez B, eds. Rehabilitación en geriatría. Barcelona: Masson, 1994;87.

Pierre J. Rehabilitación en geriatría. Barcelona: Masson, 1994;83.

Ravins PV, Mace N, Lucas MJ. The impact of dementia on the farnily. JAMA 1982;248:333-5.

Roser Solans. Alimentación por sonda en los pacientes con demencia avanzada ¿Aporta realmente beneficios? Rev Mult Gerontol 2000;1O(1):7-8.

Salom JR, López A, Saiz J, López L, Ariño C, Garay 1. La estimulación contínua en la EA y otras demencias en un Centro de Día y Rehabilitación. Geriátrica 2000; 16(9):308-14. 